\title{
Comparing Perspectives Around Human and Technology Support for Contact Tracing
}

\author{
$\mathrm{Xi} \mathrm{Lu}$ \\ Informatics, University of California \\ Irvine \\ xlu30@uci.edu \\ Hwajung Hong \\ Department of Communication, Seoul \\ National University \\ hwajunghong@snu.ac.kr
}

\author{
Tera L. Reynolds \\ Informatics, University of California \\ Irvine \\ tlreynol@uci.edu \\ Xinru Page \\ Department of Computer Science, \\ Brigham Young University \\ xinru@cs.byu.edu \\ Daniel A. Epstein \\ Informatics, University of California \\ Irvine \\ epstein@ics.uci.edu
}

\author{
Eunkyung Jo \\ Informatics, University of California \\ Irvine \\ eunkyuj@uci.edu \\ Yunan Chen \\ Informatics, University of California \\ Irvine \\ yunanc@ics.uci.edu
}

\begin{abstract}
Various contact tracing approaches have been applied to help contain the spread of COVID-19, with technology-based tracing and human tracing among the most widely adopted. However, governments and communities worldwide vary in their adoption of digital contact tracing, with many instead choosing the human approach. We investigate how people perceive the respective benefits and risks of human and digital contact tracing through a mixed-methods survey with 291 respondents from the United States. Participants perceived digital contact tracing as more beneficial for protecting privacy, providing convenience, and ensuring data accuracy, and felt that human contact tracing could help provide security, emotional reassurance, advice, and accessibility. We explore the role of self-tracking technologies in public health crisis situations, highlighting how designs must adapt to promote societal benefit rather than just self-understanding. We discuss how future digital contact tracing can better balance the benefits of human tracers and technology amidst the complex contact tracing process and context.
\end{abstract}

\section{CCS CONCEPTS}

- Human-centered computing; • Human computer interaction (HCI); • Empirical studies in HCI;

\section{KEYWORDS}

Contact tracing, Public health, Personal informatics, Self-tracking, Crisis informatics, COVID-19

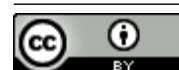

This work is licensed under a Creative Commons Attribution International 4.0 License.

CHI '21, May 08-13, 2021, Yokohama, Japan

(C) 2021 Copyright held by the owner/author(s).

ACM ISBN 978-1-4503-8096-6/21/05

https://doi.org/10.1145/3411764.3445669

\section{ACM Reference Format:}

Xi Lu, Tera L. Reynolds, Eunkyung Jo, Hwajung Hong, Xinru Page, Yunan Chen, and Daniel A. Epstein. 2021. Comparing Perspectives Around Human and Technology Support for Contact Tracing. In CHI Conference on Human Factors in Computing Systems (CHI '21), May 08-13, 2021, Yokohama, Japan. ACM, New York, NY, USA, 15 pages. https://doi.org/10.1145/3411764.3445669

\section{INTRODUCTION}

With COVID-19, the world has experienced an unprecedented global health crisis. Isolation, social distancing, contact tracing, and testing are key strategies to contain the virus prior to the release of vaccines $[2,14,68,74]$. Especially considering COVID-19's relatively long-term nature, experts believe people will need to cope with the pandemic for some time even if a safe and effective vaccine is developed [52]. A number of digital technologies have been deployed to help contain the spread of COVID-19, such as digital contact tracing, symptom trackers, and artificial intelligence (AI) chatbots for pre-screening [57, 68]. Many countries have used digital contact tracing to help contain the spread of the virus since an estimated 30\% [13] of people with COVID-19 are asymptomatic, and these individuals are frequent spreaders of the disease [2,14]. Digital contact tracing aims to identify, notify, and monitor close contacts, while human tracers typically perform the same function by directly talking to confirmed cases and their close contacts. While the term digital contact tracing often refers to tools and applications (apps) used to monitor people's location and proximal interactions [34], technology used during contact tracing often incorporates self-tracking features like daily symptom reporting. Some apps additionally enable reporting a confirmed case [46] or identifying close contacts.

Contact tracing strategies for COVID-19 have varied substantially by country. For example, South Korea has developed a contact tracing program which integrated GPS phone tracking, CCTV footage, and credit card transactions [75]. Australia [29] has developed an app which leveraged Bluetooth technology to record and identify close proximity contacts. At the beginning of the disease 
outbreak, researchers and public health authorities in many countries developed symptom trackers for individuals to self-report cases to understand the spread of the virus, such as COVID symptom study applications in the United States (U.S.) and United Kingdom (U.K.) [28]. These countries also often employ human contact tracers to augment the tracing process [57]. While most states in the U.S. relied solely on human contact tracing early in the pandemic, Virginia became the first U.S. state to publish a voluntary statewide contact tracing app, which was downloaded 300,000 times in its first week [30, 83]. As of December 2020, 19 U.S. states, as well as Washington, D.C., Puerto Rico, and Guam have developed their own contact tracing apps [22].

Human and digital contact tracing approaches have each shown strengths and weaknesses during the COVID-19 pandemic. While human tracers are able to provide emotional reassurance and personalized help, human efforts are inherently burdensome, and the speed and scale at which the measures need to be deployed under crisis can lead to inaccuracy [9, 38, 62]. Since most human tracers are trained during public health crises, errors such as missing or mis-recorded information and conveying wrong messages to confirmed cases or close contacts are inevitable [59, 78]. On the contrary, digital contact tracing can effectively mitigate some of these challenges, but also raise concerns about trust, privacy, and digital equity $[9,42,65]$. The rapid development and deployment of digital contact tracing in response to crisis runs the risk of not following best practices around privacy and security and can be counterproductive. Norway shut down its contact tracing app to avoid collecting unnecessary data from its citizens [47]. Singapore allows the police to access data collected from its national contact tracing app to investigate crimes, raising people's concerns about their privacy [72]. To understand the distinct strengths and weaknesses and their impacts, we asked the following research questions: 1) How do people perceive the respective strengths and weaknesses of human contact tracing and digital contact tracing in regions with high incidence of COVID-19? 2) How does the contact tracing strategy influence people's willingness to share information often collected during contact tracing?

Answering these questions helps extend our understanding of how self-tracked data can be leveraged in public health crises, building on literature in Crisis Informatics [32] and Personal Informatics [58]. Public health crises pose unique challenges to Crisis Informatics because there is considerable uncertainty when scientific knowledge about a novel disease is developing [40,44]. While most studies in Personal Informatics for public health have focused on understanding community-level health behaviors [5, 43, 60, 70], in the context of public health crises, it is crucial to investigate how to effectively collect individual health information to aid in efforts to understand and contain diseases.

We therefore deployed a mixed-method survey with 291 responses in the U.S. to investigate how individuals perceive human tracing and tracing technology at three different contact tracing touchpoints: identifying close contacts, being notified of potential exposure, and monitoring daily health status. We also explored respondents' willingness to share types of personal information for tracing under each strategy. We focused on the U.S. context because the high rate of COVID-19 cases at the time of study emphasized the need to understand the impact of different contact tracing approaches. Prior research on personal data privacy and crisis informatics technology have similarly taken the approach of focusing on a single country, such as the U.S. [63, 87].

Collectively, participants did not express a preference towards human contact tracing or digital contact tracing, seeing each having strengths and weaknesses. People perceived digital contact tracing as more effective for promoting privacy, convenience, and data accuracy, while human contact tracing offered benefits around data security, emotional reassurance, advice, and accessibility. Participants particularly preferred digital contact tracing when monitoring daily health during self-quarantine, as the need for repeated entry increased the importance of convenience and accuracy. Respondents felt more comfortable with the idea of sharing some personal information, such as identifying information and demographic risk factors, with human tracers.

This study provides the following contributions to HCI, Crisis Informatics, and Personal Informatics.

- An understanding of people's perceived preferences between human contact tracing and digital contact tracing, finding that people perceive each approach to have benefits and risks respectively. People perceive technology as more convenient and accurate, while human tracers seem more effective for providing advice and support.

- An understanding of how different phases of contact tracing and types of data required to be shared influences people's perceptions of human and digital contact tracing approaches. Although people consider technology more convenient when reporting symptoms, which requires repeated engagement, they prefer disclosing sensitive information related to their identities to human tracers.

- Design recommendations to balance the benefits and perceived concerns towards human and digital contact tracing approaches, such as flexible and hybrid systems. We suggest that these systems can go beyond public health benefit to provide individual benefit as well, increasing the personal value of contact tracing and keeping people engaged in the process. We also identify the need for systems to convey authority and transparency to help gain trust.

\section{BACKGROUND: COVID-19 AND CONTACT TRACING}

COVID-19 presents the biggest worldwide public health crisis in the past 100 years [2]. The virus that causes COVID-19 has infected over 22 million people in the U.S. and 90 million people globally through January 2021, causing over 374,000 deaths in the U.S. and 1.9 million deaths globally [35]. It is highly contagious, transmitting more quickly than previous viruses, with estimates suggesting that one in five individuals would be at risk of being infected if spread is uncontained [26].

Contact tracing has been recommended by public health authorities such as the World Health Organization (WHO) and U.S. Centers for Disease Control and Prevention (CDC) as a major measure to prevent disease transmission [26]. Contact tracing involves monitoring people who likely have COVID-19 based on their symptoms or who have been confirmed to have COVID-19 through a positive laboratory test (positive cases) and then identifying individuals 
with whom they were within 6 feet for at least 15 minutes (close contacts) [26]. Close contacts are then notified and required to selfquarantine for 14 days to watch for the development of symptoms and to prevent further disease spread. Contact tracing has been proven to be effective for containing previous disease outbreaks, such as Severe Acute Respiratory Syndrome (SARS) in 2003 [59], Middle East Respiratory Syndrome (MERS) in 2015 [65], and the Ebola Virus in $2014[9,69]$. Contact tracing is particularly crucial for slowing the spread of COVID-19, as this virus is often transmitted before people show symptoms $[2,61]$.

According to U.S. CDC $[17,24]$, the contact tracing process involves three major touchpoints: (1) Identifying: a confirmed positive case is contacted by the public health department to check their health status, such as COVID-related symptoms. They are also asked to identify with whom they have been in close contact by disclosing where they have been and who they have interacted with in the past 14 days. (2) Notifying: local public health departments contact people who the confirmed case has been in close contact with to let them know of the potential risk of exposure without disclosing the identity of the positive case. The contact is asked to self-quarantine for 14 days. The contact is also asked to share with whom they have been in close contact and where they have been to investigate if there are other potential close contacts. Contacts also share other personal information, such as risk factors and accessibility to necessary resources, in order to get instructions and support services, such as food and medicine [15]; (3) Monitoring: both the positive case and all contacts monitor their own health, such as body temperature and COVID-related symptoms, and share it with the local public health department during the 14-day self-isolation or self-quarantine. The contacts may be able to take a COVID-19 test depending on availability in their area, but the U.S. CDC still asks them to self-quarantine for 14 days regardless of whether the result is positive or negative [24].

Across these three touchpoints, the approaches that public health organizations have used for contact tracing in COVID-19 can be grouped into two major categories: using human tracers and using digital contact tracing. Human tracers are individuals who have been trained by public health authorities to identify confirmed cases, notify close contacts, and monitor the cases and their close contacts during their 14-day self-isolation or self-quarantine [15]. At all touchpoints, the human tracers typically call the positive case or close contact and manually log all information they provide into the public health department's database, such as close contacts, daily temperature, and related symptoms. Digital contact tracing usually refers to digital proximity and exposure notification tools that track real-time location information and/or close proximity encounters to identify and notify close contacts, and monitor symptoms of cases or close contacts during self-isolation or self-quarantine [17]. In some digital contact tracing approaches, positive cases can selfreport a test result and securely report close contacts via a digital platform (e.g., a mobile app or a website).

Human tracers and tracing technology have each revealed advantages and disadvantages while being implemented to address COVID-19. Human tracers can provide emotional support for cases or contacts and prioritize people's privacy [9]. Human tracers typically provide empathy, listen to people's stories, and comfort people by providing informational guidance [59, 78]. However, locales have faced challenges implementing human contact tracing, such as being unable to train contact tracers quickly enough to respond to outbreaks $[9,12,38,59,62,78]$. On the other hand, studies in countries such as South Korea have demonstrated that digital contact tracing can be effective for containing the spread of diseases $[38,65]$.

Several studies have pointed to challenges and limitations of technology-based approaches. For example, digital contact tracing introduces privacy concerns, raising discomfort with information about people's identity and whereabouts will be tracked and disclosed [65]. The location and proximity aspects of digital contact tracing can only be effective when a high portion of a locale has adopted or enabled the approach [8] with some studies suggesting adoption levels of $56 \%$ to $95 \%$ are required to be effective [9]. This participation requirement presents a tension between protecting people's rights by giving them the choice to adopt and the need to increase the effectiveness of the automatic contact tracing approach [85]. Meanwhile, digital contact tracing has raised concerns around digital equity as certain populations particularly vulnerable to the COVID-19-such as older adults, people who are homeless, and socioeconomically disadvantaged individuals-are less likely to have access to a smartphone and benefit from the use of digital contact tracing [9]. In addition, research has highlighted the need to consider the potential stigma that positive cases may face if their demographic information is leaked when using a digital approach $[42,53]$.

As of January 2021, the use of human contact tracing and digital contract tracing has varied substantially by country and locality. In the United States, the CDC has recommended that human contact tracing be used as the main approach. As a result, public health departments in each state have implemented their own procedures and programs to train and utilize teams of human tracers. For example, California first adopted a statewide contact tracing program run by the public health department and aims to train 20,000 tracers (including paid and volunteer tracers). Later in December 2020, California launched its statewide exposure notification system [22]. Indiana partnered with a private crowdsourcing company to hire and train human tracers [21, 77]. Meanwhile, some states and counties have focused more on utilizing technology, such as case management tools and symptom trackers, to augment the work of human tracers [26, 62, 77]. Virginia became the first U.S. state to publish a statewide contact tracing mobile application [30, 83], and 19 U.S. states are using a mobile solution as of December 2020 [22].

Outside of the U.S., countries such as South Korea, Singapore, India, and Australia have developed and deployed nationwide digital contact tracing. Both Australia [29] and Singapore's [71] mobile applications leverage Bluetooth to measure proximity between devices and record encounters. India's contact tracing application uses both Bluetooth and GPS to trace people's close contacts and previous location history [1]. Using the contact tracing applications are voluntary with a few exceptions (e.g., mandatory for immigrant workers in Singapore, citizens living in containment zones, and government employees in India) [1, 25, 29]. South Korea has adopted a more comprehensive contact tracing strategy that integrates data from CCTV footage, credit card or debit card transactions, and GPS phone tracking, to monitor people's whereabouts and actions and automatically notify positive cases via text message $[65,75]$. 
Most contact tracing apps are voluntary and opt-in. While the ideal adoption rate is between 56 and 95\% [9], the actual adoption rate of many countries with national-level contact tracing apps is closer to $10 \%$ [27].

\section{RELATED WORK}

Digital contact tracing for public health events relates to prior literature on technology use during crisis events and personal tracking technology in health domains.

\subsection{Crisis Informatics}

Crisis informatics has examined the relationship between technologies, individuals, and organizations under crises such as natural, technical, anthropogenic, and criminal hazards [32]. It has explored how people leverage technology to solve problems during crises. Popular strands of research include examining how individuals voluntarily exchange information online to keep each other informed of the current situations during crises [44, 45, 76], how emergency service workers utilize multiple communication channels to effectively share information $[18,79]$, and how authorities communicate and distribute information to the public during crises [32, 64, 82, 87].

Crisis informatics has mainly focused on natural disasters (wildfire, flood, and hurricanes) and criminal acts (bombing and other mass violence events). Understanding how citizens use technology to cope with public health crises such as major disease outbreaks has rarely been studied [44]. However, public health crises pose unique challenges because they bring about considerable uncertainty while scientific knowledge about a novel disease develops, especially at their beginning. This lack of scientific knowledge could lead to ambiguity about affected spatial areas, potential risks to individuals, and the best strategies to prevent the spread of the diseases [40,44]. On the contrary, risks in manmade and natural disasters, such as bombings and tornadoes, are easier for the public to see than the impact of public health crises like viruses [45].

In response to outbreaks in recent years (e.g., SARS in 2003, Avian Influenza in 2006, H1N1 in 2009, Ebola in 2014, Zika in 2015, COVID-19 in 2020), digital technology has increasingly been implemented and applied for managing, tracing, and processing large scale data [86]. Besides digital contact tracing, other technology such as big data and AI technology has also assisted in predicting and managing the spread of COVID-19 [84]. Other examples of technology developed to help manage pandemics include digital screening technologies such as sensors that measure people's temperature in public space, and telemedicine and virtual care platforms [84].

\subsection{Personal Tracking for Public Health}

Personal informatics studies how technology can help people collect personal data through self-tracking, analyze and reflect on collected information, and take further action [58]. The HCI community has largely considered self-tracking for the primary purpose of self-improvement such as promoting a healthier lifestyle [55], but research has increasingly examined how to support collaborative and shared tracking among peers or families or between patients and providers [36]. Digital contact tracing is a self-tracking process that aims to benefit not only individuals, but also society as a whole during public health crises.

Studies are increasingly exploring how tracked data can be used to further public health knowledge [5, 43, 60, 70]. Prior studies have leveraged large-scale aggregated self-tracked data to understand the community level's health status and health-related habits. For example, Lim et al. use data mining and clustering to analyze the daily steps of hundreds of thousands of citizens to understand cyclic patterns in people's walking habits [60]. Althoff et al. [5] used physical activity data from people in 111 countries and found that cultural inequities, such as reduced activities in females compared with males, can help predict obesity prevalence. Community-level self-tracked data can also be useful for understanding the effectiveness of public health interventions. For example, Gordon et al. [43] analyzed 1.4 million MyFitnessPal users and their weight-loss goals to explore the influences of self-tracking apps' goal setting and achieving aspects, revealing that whether people could successfully reach their goal is closely related to their weight-loss behaviors in the first 7 days. Similarly, Shameli et al. [70] performed a large-scale analysis of daily steps from a mobile app's walking challenges to examine the influence of gamification design on people's behaviors.

There has been little research exploring how to leverage technologies to engage the public in contributing to disease surveillance and response. However, there are notable exceptions focusing on the benefits for public health versus people's experience of using the technology. For example, the Flu Near You project asks people to submit a weekly online or app-based report on whether or not they have experienced influenza symptoms in the last week [73]. In a comparison of multiple methods for influenza surveillance, Baltrusaitis et al. [7] found that, with sufficient participation, Flu Near You corresponded well to trends in data from official sources (e.g., electronic health record). In addition, Freifeld et al. [39] used mobile phone technology to enable the public to submit reports related to their health or the health of their community, such as infectious disease-related school closures. Leveraging publicly-available data, some health departments have also created dashboards to identify social media posts with indicators of foodborne illness, following up with these social media users for further details [48]. Unfortunately, there is a paucity of research on the public's perceptions of these technologies.

\section{METHODOLOGY}

We conducted a survey to understand people's perceptions towards the respective strengths and weaknesses of human contact tracing and digital contact tracing in the complex contact tracing process in August 2020, after a pilot.

\subsection{Survey Structure}

We iteratively developed and tested the survey through revision among the authors, feedback from other members of our research group, and a pilot deployment on a research-focused crowdsourcing platform named Prolific [66]. Apart from the consent to participate, the questionnaire contains three main sections. We first offered an introduction of contact tracing to explain the goal and the roles of both human contact tracing and digital contact tracing. Our survey questions included a mix of Likert-scale and open-ended questions 
Table 1: Demographic breakdown of our survey respondents, recruited from a representative sampling service.

\begin{tabular}{lllll}
\hline Gender & Age & Race & Educational background & Annual household income \\
\hline Female: $146(50.17 \%)$ & 18-27: $46(15.81 \%)$ & White: $187(64.26 \%)$ & Less than high school: $1(0.34 \%)$ & $<=\$ 35 \mathrm{~K}: 101(34.71 \%)$ \\
Male: 141 (48.45\%) & $28-37: 57(19.59 \%)$ & Black: 35 $(12.03 \%)$ & High school: 32 $(11.00 \%)$ & $\$ 35 \mathrm{~K}-\$ 50 \mathrm{~K}: 45(15.46 \%)$ \\
Non-binary: 2(0.69\%) & $38-47: 46(15.81 \%)$ & Multiracial: $27(9.28 \%)$ & In college: $90(30.93 \%)$ & $\$ 50 \mathrm{~K}-\$ 75 \mathrm{~K}: 43(14.78 \%)$ \\
& $48-57: 51(17.53 \%)$ & Asian: 20 $(6.87 \%)$ & Bachelor degree: $90(30.93 \%)$ & $>=\$ 75 \mathrm{~K}: 88(30.24 \%)$ \\
& $58+: 83(28.52 \%)$ & Latino: $10(3.44 \%)$ & In graduate school: $10(3.44 \%)$ & \\
& & Graduate degree: $64(21.99 \%)$ & \\
\hline
\end{tabular}

to understand respondent's perspectives on contact tracing. The survey concluded with optional demographic questions and questions to understand people's perceptions of COVID-19 drawn from public opinion surveys $[16,81]$. We preferred optional demographic questions in case participants were not comfortable with disclosing some of their demographic information. The survey was classified as exempt by our institution's IRB since the survey methodology does not involve more than minimal risk to participants and no identifiable information was collected during the study.

Our questions asked participants to evaluate their thoughts on contact tracing at the three main touchpoints identified in our related work section: helping identify close contacts, being notified as close contacts, and monitoring and sharing health status during a 14-day self-quarantine. After a brief description of each touchpoint, we asked participants to rate their preference towards human contact tracing or digital contact tracing on a five-point Likert scale question: "How comfortable do you feel about the following approach (human contact tracing or digital contact tracing) of helping identify close contacts/being notified/monitoring and sharing daily health status?" We further asked participants to describe the potential benefits and risks of each of these two approaches for each touchpoint through open-ended questions. Participants also answered a five-point Likert scale question of their willingness to share different types of personal information that are typically collected by one or both approaches during the contact tracing process (e.g., personal risk level due to demographics, location history, close contacts). The survey concluded by having participants indicate and explain which approach they preferred for contact tracing overall. The survey took approximately 15 minutes to complete.

\subsection{Recruitment}

We created our survey on Qualtrics [67] and recruited survey responders on Prolific [66], a research-focused crowdsourcing platform, in August 2020. We used Prolific's representative sampling service to recruit 300 people who live in the U.S. whose age, gender, and racial backgrounds approximately represent the demographics of the country as Prolific stratified our sample size across age, gender, and racial background. We paid each participant $\$ 2.38$ for responding based on Proflic's suggestions for a 15-minute survey. We excluded nine responses from people who took less than three minutes to answer the survey and ended up analyzing 291 complete responses.

Table 1 shows how participants self-identified demographics. Participant ages ranged from 18 to 77 ( mean=45.0, $s d=15.5$ ). 286 (99.65\%) of participants' education level was high school or higher, and $164(57.14 \%)$ had at least a bachelor's degree. The median annual household income of our participants fell between $\$ 50,000$ and $\$ 75,000$ annually. Participants came from 44 of the 50 U.S. states. Six participants had been in contact with public health authorities as a positive COVID-19 case or a close contact.

\subsection{Analysis}

We analyzed both closed and open-ended survey questions to understand how participants perceived the strengths and weaknesses of human contact tracing and digital contact tracing in specific contexts as well as holistically. We quantitatively compared people's expressed comfort with the two contact tracing approaches for the three touchpoints using mixed-effect ordered logit models. We treat the comfort level Likert ratings as an ordinal response. We treat the fixed effects as categorical, examining the contact tracing approaches (two levels), touchpoints (three levels), and interactions between them. Analysis of comfort level with sharing data types similarly treated contact tracing approach and datatypes as categorical fixed effects. We treated participant IDs as a random effect to account for variations in use of the scales. We corrected for multiple comparisons in post-hoc tests with false discovery rate corrections. This method has a lower false positive rate, but higher false negative rate compared to Bonferroni corrections.

We thematically analyzed [10] the open-ended responses. Two authors first individually read and open coded 30 survey responses, meeting to generate a codebook and iterating on the codebook with the rest of the research team. The authors gave formal definitions and examples for each subcode, holding meetings to discuss the codebook and definitions to reach consensus. The final codebook contained 6 parent codes (trust, emotion and feelings, communication, privacy and data security, data quality, and accessibility) and 24 subcodes in total. Two authors used the codebook to code a different set of 30 surveys, reaching initial agreement of $80 \%$ across codes and discussing to resolve ambiguities. The first author then coded the remaining survey responses. We quote participants according to participant ids (e.g., PXX). Since we required respondents to complete all questions except demographics, the denominator of qualitative interview questions is 291 .

\subsection{Limitations}

Though digital contact tracing has been adopted in many countries, we focused on exploring how people perceive contact tracing and its two different approaches in the United States. COVID-19 case counts in the U.S. were among the highest in the world during 
deployment of the survey (both absolute and per capita), so understanding perceptions in the U.S. can help inform perceptions of human and digital contact tracing approaches when need for tracing is high. Because digital contact tracing was not adopted as part of a national strategy at the time, adoption was lower than in other countries and the implemented procedures vary across states.

We ran several rounds of testing before launching the survey to ensure the structure and wording was interpretable and reliable, including among lab members and on Prolific. When analyzing our final survey responses, we noticed a few respondents who expressed confusion or misunderstanding about how human and/or digital contact tracing would work in practice. For example, they thought that the human tracer would contact the positive case or the close contact in person (e.g., visiting them at home to ask about who they had been in contact with). Such misunderstandings, particularly when reinforced by media surrounding the virus or contact tracing, may have influenced perceptions of tracing strategies among some participants.

Although we aimed to recruit a sample which represents the demographics of the U.S., our sample was not as representative as we expected on a few key dimensions. Compared with the U.S census bureau's data [80], our survey underrepresented people who self-identify as Hispanic/Latino. Participants' education levels were higher than the country as a whole, which may correlate with higher technology acceptance or higher perceptions of the risk of the virus. Because most of our participants had not experienced contact tracing firsthand, their responses primarily drew from their perceptions on contact tracing from the media at large, as well as our introduction and description of contact tracing and its two approaches in different touchpoints provided in the survey. Our results may also not be generalizable to countries with different strategies or under different circumstances, such as those that include digital contact tracing in their national response strategy, where using the technology may be required, or where case counts are low. The application of digital contact tracing may also be shaped by complex socio-cultural contexts in different countries, such as greater privacy concerns in Western countries [51], the political role that contact tracing apps play in helping the government reopen society [20], and experiences obtained in previous pandemics [65].

We asked participants to describe why they felt comfortable or uncomfortable with each approach to contact tracing under each touchpoint using open-ended questions. Our qualitative analysis aimed to explore the variety of reasons why people prefer human tracers or tracing technology, but future work is required to better understand the relative prevalence of such reasons among people in the United States. Through our qualitative coding, we counted the number of participants who expressed their perspectives, but the participant counts should not be treated as percentage agreement. Instead, the participant counts are indication of the prevalence of first thoughts that came to participants' minds upon being asked (e.g., primary benefits or drawbacks of tracing strategies under different circumstances). We expect that more participants may have agreed with a given benefit or drawback if explicitly asked.

\section{RESULTS}

Overall, participants felt concern about COVID-19 on a personal level and were overall comfortable with the concept of contact tracing. In our survey, the majority of participants agreed that COVID-19 is a threat to the health (U.S.: $85.6 \%$; Self: $74.9 \%$ ), economy (U.S: $93.1 \%$; Self: $69.8 \%$ ), and daily life (Self: $82.5 \%$ ) of both the U.S. as a whole and individuals. Most participants described themselves as "very closely" (39.0\%) or "fairly closely" (45.6\%) when being asked how closely they have been following news about COVID-19, while only $12.2 \%$ indicated that they did not pay close attention, and $3.1 \%$ said that they did not follow news about COVID-19 at all.

Overall, participants were comfortable with the idea of contact tracing (Figure 1), with more than half rating themselves as either comfortable or very comfortable in all three touchpoints with both human tracing $(54.3 \% ; 69.1 \% ; 51.5 \%)$ and digital tracing $(55.7 \% ; 65.6 \%$; $63.6 \%$ ). Twenty-one participants mentioned being willing to use either contact tracing approach as long as contact tracing could help contain the spread of COVID-19. For example, P268 said, "I think either way, it doesn't make a difference to me personally. I understand fully why we need to have contact tracing, whether that's by human or technology, we need to gather the data and find a way to contain the virus."

Participant's comfort level with contact tracing differed by touchpoints $\left(\chi^{2}(2, \mathrm{~N}=1746)=65.23, p<0.001\right)$. Participants were most comfortable with the idea of contact tracing for notifying contacts $(Z=7.47, p<0.001,95 \%$ CI $0.35-0.68$ higher on a 5-point Likert scale) and least comfortable with identifying close contacts $(Z=-6.32$, $p<0.001,95 \%$ CI $0.27-0.60$ lower on a 5-point Likert scale). However, we did not observe an overall comfort preference between human tracing and digital tracing $\left(\chi^{2}(1, \mathrm{~N}=1476)=1.53, p=0.22\right)$. We observed an interaction effect between tracing approach and touchpoints $\left(\chi^{2}(2, \mathrm{~N}=1476)=12.98, p<0.01\right)$. Although we did not observe a significant difference in comfort between tracing approaches for the first two touchpoints, identifying $(p=0.48)$ and notifying ( $p=0.41$ ), we observed an effect for the third touchpoint, monitoring. In this touchpoint, participants were more comfortable with digital tracing for reporting daily health during self-quarantine ( $Z=3.64, p<0.001,95 \%$ CI $0.28-0.93$ on a 5-point Likert scale).

Nineteen participants were opposed to the idea of contact tracing altogether. Some believed that they themselves could perform contact tracing and inform close contacts: "I will personally communicate with the people whom I have been in close proximity with in my own way and on my own terms" (P208). Some believed they had already taken enough measures in public spaces to protect themselves from being infected: "I' $m$ wearing masks and social distancing so I shouldn't be in that much danger or endangering others" (P79). Of these 19 participants, 13 rejected the idea of contact tracing specifically because "It's a complete invasion of privacy" (P271) as "Someone would still have all of my information of symptoms, where I had been and who I was in contact with" (P271). P130 pointed out that no matter whether a human or a technology was doing the tracing, the data will still be stored and entered and thus vulnerable: "A person may know my identity, and a technology would have my data stored which could be hacked." 


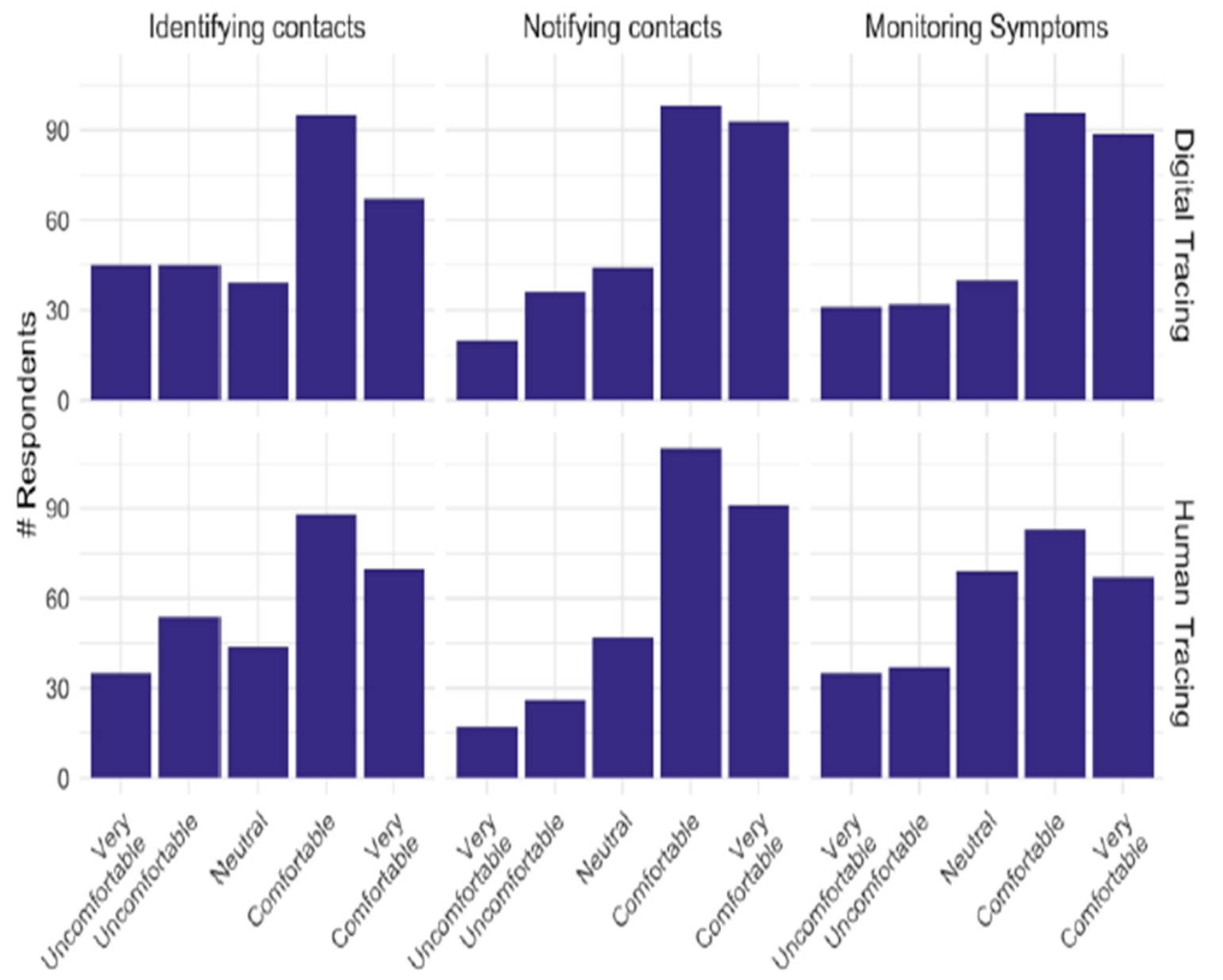

Figure 1: Participants were overall comfortable with either human tracing or digital tracing, but they preferred to interact with technology when monitoring symptoms.

\subsection{Different Perceptions towards Human and Contact Tracing}

From the qualitative analysis, we identified seven themes around participant's preferences towards either human tracing or digital tracing: privacy, security, convenience, emotional reassurance, advice, accuracy, and accessibility. The benefits and drawbacks that participants identified were relatively balanced between the two approaches, aligning with participant's range of preferences on the entire contact tracing process (Figure 1). People favored human tracing when considering matters of data security, emotional reassurance, advice, and accessibility, while favoring digital tracing when considering their privacy, convenience, and the accuracy of collected data. These preferences influenced their comfort level sharing different kinds of data often requested during contact tracing (Figure 2).

5.1.1 Privacy. Participants described privacy benefits of digital tracing, feeling that technology allowed for anonymity and could enable positive cases or close contacts to avoid being judged by human tracers for their COVID-19 status or background. Some participants also felt contact tracing with humans could bring up social anxiety, particularly around a sensitive health topic. We did not identify any significant differences between participant's comfort level with human tracing and digital tracing when disclosing where they have been $(p=0.15)$ or who they have been in contact with $(p=0.27)$. However, 11 participants felt that human tracers might judge the personal information they are required to disclose. P102 described, "If you feel embarrassed about something you have done or somewhere you have been it is easier to give the information to the contact tracing technology as it will not judge you" (P102). Thirteen participants regarded the human tracer as strangers who are "faceless" (P26) with "unexpected calls" (P48). The distrust towards human tracers makes them less willing to reveal personal information: "I don't want to talk to more strangers about my health or lifestyle" (P232). P67 particularly expressed the fear that their demographics could potentially bring up racial discrimination: "I would most likely prefer a form of contact tracing technology because 


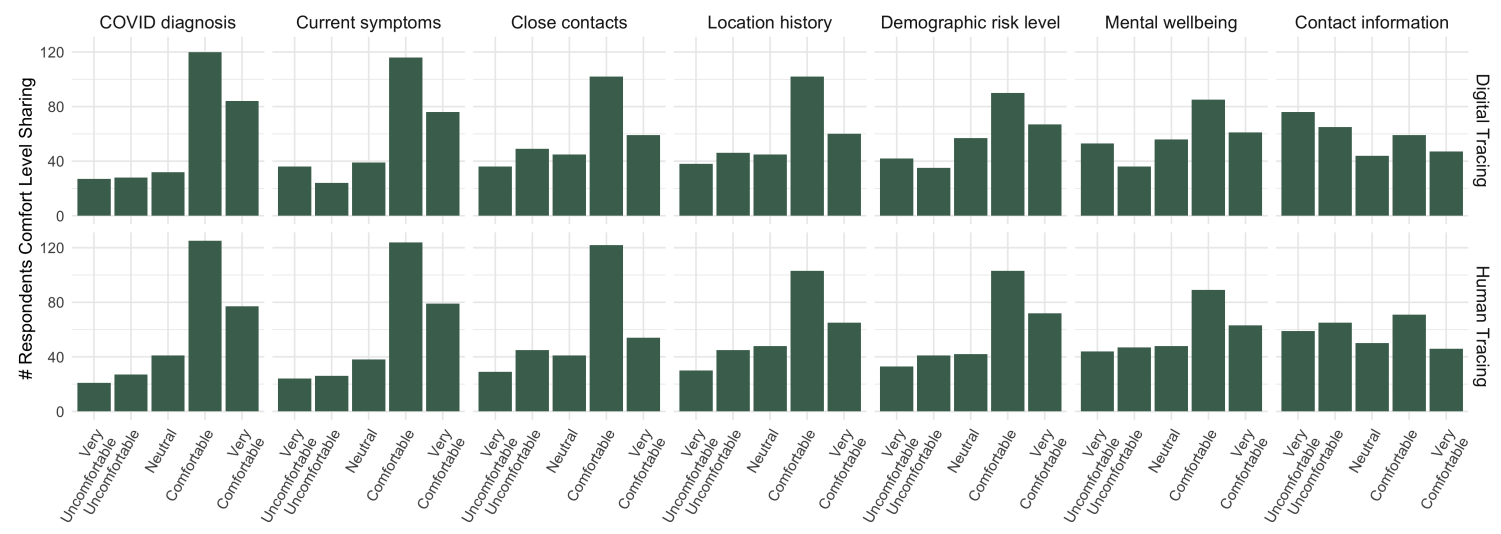

Figure 2: Overall, participants were most willing to share confirmed diagnosis and daily symptoms, while least willing to share contact information and mental health status. Participants marginally preferred sharing their daily symptoms, contact information, and demographic risk level with the human tracing.

of all the racial discrimination that has been going on especially targeting people of my race. I would not want to be subjected to any preconceived notions of humans."

Five participants worried that they would be stigmatized for being a positive case or a close contact because it indicates they did not follow best practices: "You're less likely to feel judged [about] when you might be in human interaction if you weren't being careful" (P169). P39 similarly expressed, "I feel like there is almost a stigma and it's like you are embarrassed for being around someone with covid, they might judge you for not social distancing or whatever considering the case." Participants felt technology would not judge a person for being infected or a contact. Seventeen participants felt technology could mitigate feelings of judgment and privacy invasion because "Anonymity seems to be the deciding factor" (P288) As P165 said, "I would also like the idea that the information was anonymous - contacts could be notified without revealing who the positive case was. I feel like there is too much judgment in our society for those who come down with the disease."

Some participants felt interacting with a human tracer could trigger emotional anxiety, either because they self-described as introverted (6 participants) or as sensitive about human interaction (16 participants). For example, both P132 and P264 expressed their struggles of communicating with people via phone or video call because of being shy: "I am personally quite shy and introvert and hate phone or video chats" (P132), "I'm shy and struggle with phone conversations or zoom meetings so I wouldn't be able to converse properly. I would often mess up my words and it would be difficult for me and for the interviewer" (P264). Some participants felt human tracing could raise their anxiety: "Some people wouldn't feel comfortable talking to someone, me for example, I have anxiety pretty bad so $i$ wouldn't want to do a face to face or even voice." (P111). P130 imagined how technology could relieve the stress brought by a human tracer: "It would likely be less stressful, and I'd have time to think without the pressure of someone else being on the other end of the phone waiting for an answer."

Although most participants thought technology could better preserve privacy, six believed human tracers would be more effective in establishing authority. P144 felt, "human contact tracers trained by public health officials would be more trustworthy." Similarly, P54 thought technology might not have this weight, describing technology as "unverified by a doctor's office or some kind of medical authority." Some participants believed that they had "more control over the exact amount of actual information that they receive" (P205) when interacting with a human tracer because "I have the ability to give them the information that I want to share. If I don't want to share my mental state, then I have that choice" (P226).

5.1.2 Security. Participants were overall less willing to share data with contact tracing technology than with a human tracer $(Z=-$ 3.37, $p<0.001,95 \% \mathrm{CI}=0.09-0.35$ lower on a 5-point Likert scale). Moreover, when sharing identifying information, participants were marginally more willing to share it with the human tracer $(Z=1.84$, $p=0.066,95 \%$ CI 0.02 lower- 0.66 higher on a 5-point Likert scale). Participants' unwillingness to share data with contact tracing technology was often due to concerns around data security. Compared with human tracers who participants felt were typically "trained by public health officials" (P144), participants often associated contact tracing technology with the technology companies who developed them or the government at large: "vast government/business database" (P160). Therefore, six participants worried about information being intentionally or accidentally leaked, and eight worried that their data would be used by a third party. For example, P61 believed the database of a mobile application had more of a possibility of being hacked than information in a public health department's database: "app data is more frequently attacked than direct medical records, information leaked could violate HIPPA[sic] and privacy." Participants felt technology, especially mobile apps, could easily cause information leakage: "The computer program is just too dangerous as a basis of leaking information." (P205) and "data information and personal information can be hacked over the app" (P241). Other participants believed the data collected by technology would be shared with companies looking to use it for profiling or marketing: "My only issue would be having full trust that the app will not take the data and then sell it for a profit or use it for other purposes it was not 
originally intended for" (P185). Others felt more generally reluctant to "give my information to the government to use" (P4).

5.1.3 Accuracy. Sixteen participants felt digital tracing had greater ability to capture data more accurately and account for potential challenges around human entry. Ten participants brought up that some information, such as location history and close contacts, is potentially easier to be traced by technology: "Assuming this would be based solely on location tracking and whether I have a positive/negative diagnosis, this would make more sense to have it be automated and done via GPS" (P34). Six participants also thought that technology could help prevent human errors because human memories are limited, especially making identifying contacts challenging: "Unreliable information from a person if their memory is not the best, if they forgot places they have been, or if they forget people, they have been in contact with due to being distracted or diseases such as dementia. Not getting exact routes of movement" (P53).

Five participants worried that technology would be ineffective because it would not achieve high enough adoption, even if they preferred technology themselves: "It's likely to 'catch' lots more potential community-spread exposures than a human contact tracer might, provided enough people adopt the technology. The downside to the tech solution is that it requires a majority of the population to adopt it in order to be effective" (P31). P151 also expressed a similar concern: "The only drawback I could see is if others do not use the app." This concern echoes prior studies' expectation that digital tracing can only be effective when $56 \%$ to $95 \%$ of the population opt-in $[8,9]$. Conversely, two participants felt that people would be more likely to provide accurate information when interacting with a human tracer than with technology: "there is more accountability and reminders to tell the truth if someone is relying on you" (P21).

5.1.4 Convenience. Sixty-three participants felt that technology could save them effort, with human tracing potentially introducing interaction burdens. Participants envisioned that it would be burdensome to interact with human tracers because they are either busy or they do not want to communicate with human tracers. This was particularly prevalent when considering providing data in the monitoring symptoms touchpoint since both positive cases and close contacts typically share daily health status during self-quarantine. Participants preferred using technology in this touchpoint $(Z=3.64, p<0.001,95 \%$ CI $0.28-0.92$ higher on a 5-point Likert scale). Four participants described themselves as "very busy" (P69) and they worried that communication with a human tracer could conflict with their working hours: "have to worry about phone calls especially during working hours" (P147). As people have to self-monitor and share health status on a daily basis during selfquarantine, eight participants felt that communicating with a human tracer everyday would be a burden because they would " $h a v[e]$ to go back and forth with a human" (P2) and "call someone almost every day." (P90). Additionally, seven participants described that they were more willing to interact with the technology because "the efficiency of digital tracing would slightly outweigh" (P197) the human tracer. Four participants believed that technology could remove the burden of interacting with human tracers by enabling people to enter data at their own convenience: "I can report all of my data on my own time through an app" (P136).
Participants felt technology would offer convenience by tracking data passively (7 participants) and enabling data to be managed at scale (5 participants) when tracking and notifying people. Participants valued automation when helping identify close contacts because: "The process would be almost entirely automatic and require little effort from me. It sounds super efficient" (P213). Comparatively, they felt it would require more effort to try to remember when being interviewed by a human tracer: "It's very fallible, as I would have to recall everywhere I've been and, if possible, who I've been near. This sounds very difficult and stressful to try to do" (P213). Participants were also concerned about controlling the spread of the virus as quickly as possible, feeling that digital tracing was better-suited to track data from a large number of people: "monitor more people in a shorter amount of time." (P85). Participants also felt that it would be easier to inform people through digital tracing, such as $\mathrm{P} 4$ "it could notify mass amounts of people at once and help stop spread", and P110 "I would like the ability to be notified immediately and easily share my own proximity if I have been infected." However, five participants felt human tracing could be more convenient, perceiving a high burden in entering information using technology: "I don't really feel that I'd prefer a human. But an app would require more typing for me." (P284). Technology also could add difficulty if someone needs additional help: "can provide quick information without having to click on a bunch of links or go to other sites" (P38).

5.1.5 Emotional Reassurance. Fourteen participants felt human tracers could empathetically understand the negative feelings that positive cases or close contacts with COVID might experience, carefully convey bad news, and help relieve negative feelings. Participants mentioned how stressful they thought it would be to be a positive case or be notified as a close contact: "I will get anxious and distress thinking that I probably infected other people" (P186). In this situation, participants felt that emotional reassurance from human tracers would be particularly helpful because the human tracer could understand the challenges that people are experiencing: "Humans can feel empathy and emotions for people who have that disease" (P75). P264 felt similarly: "Also the human empathy and compassion can be comforting to someone who is under stress." Participants pointed out that human tracers are more able to be aware of people's emotions even just by hearing their voice: "They can tell by my voice how I am feeling (Concerned, frightened, etc) and can say things that would comfort me" (P267). P273 felt that human tracers were well-suited to conveying bad news to people because they are trained: "It's always reassuring to hear bad news from another person trained to give it." Although many participants feared a human tracer would judge them for being a positive case or a close contact, P206 believed that an empathetic human tracer could help relieve the shame of having a positive diagnosis: " $A$ human tracer could lend a sympathetic ear and make the subject feel more comfortable about sharing. He or she could walk the subject through the steps and minimize any feelings of shame held by the subject."

5.1.6 Advice. Participants imagined that if they were diagnosed as a positive case or identified as a close contact, they would require further information about how to act. P50 felt they would want to " ask questions and get verifications on things you do not understand or even ask what the next stage was. if they do test positive." Forty-one 
participants favored human tracing because they felt the approach would enable an interactive conversation. P88 expressed that they "would like to have the ability to ask questions about what's next," and P104 added "I think I would like to have the human interaction about this situation and ask questions about the situation. Especially if I am going to be quarantining for 2 weeks. I would like to be able to get more information." Besides answering questions, eight participants felt human tracers would be better equipped for providing instructions and connecting people with resources: "advise the person on how to get the appropriate medcare for the treatment" (P176). This aligns with federal guidelines that when being notified, contacts will receive information about risk factors and accessibility to resources [15]. Participants marginally preferred to share their demographic risk factors for COVID-19 (e.g., age, lung condition) with a human tracer compared to a technology $(Z=1.77, p=0.076,95 \% \mathrm{CI}=0.03$ lower-0.63 higher on a 5 -point Likert scale).

Overall, participants were skeptical that technological contact tracing solutions would be able to offer advice. Participants believed that they would need to spend extra effort to get necessary information and assistance via technology because "You can't directly ask the text/voicemail any questions and have to try to get ahold of a real person if you do have questions" (P38). They also questioned technology's ability to provide personalized guidance to answer their specific questions: "not able to ask for more information or get a personalized plan for what to do next" (P45).

5.1.7 Accessibility. Seven participants worried about issues of digital equity because many people lack access to the internet or mobile phones. Even among people who can access mobile phones, participants felt that it could still be a challenge for certain groups of people, such as older adults, to interact with technology. Overall, younger participants tended to prefer digital tracing more than older participants $(Z=4.00, p<0.001,95 \%$ CI $0.01-0.04$ points on a 5-item Likert scale per year older). P13 felt that "information gaps" may be caused by the lack of access to technology and the inability to interact with technology, making older adults at more risk. P86 described that technology "only works for people who have phones and the ability to use apps, which leaves out (some of) the elderly, the poor, the homeless, immigrants, and some of the disabled. Arguably the most vulnerable of populations." P256 expressed a similar concern: "many people do not have access to telephones or internet access and are likely to already be an at-risk group, so this is a dangerous method because it leaves out this population." By contrast, participants felt that human tracing might be more flexible. P52 felt that human tracers "would be able to work around issues that come up on a case by case basis (i.e. if someone is homeless and has no address, they can use a P.O. Box or other contact)."

Meanwhile, technology could help increase accessibility for some individuals with disabilities to be engaged in the contact tracing process. Two participants with hearing issues or speech impediment expected that technology would better facilitate their own participation in contact tracing since they have difficulties interacting with people. $\mathrm{P} 233$ described that they "have auditory processing issues and I really struggle to talk on a phone, and some voices are worse than others." P22 similarly felt it would be easier to express themselves to contact tracing technology because "I have a speech impediment so it would be much easier for me to enter information in on an app."

\subsection{Contact Tracing in Three Touchpoints}

Participant's overall perceptions influenced their comfort levels with human and digital contact tracing approaches at the three touchpoints.

5.2.1 Touchpoint 1: Identifying. In the first touchpoint, where positive cases help identify potential close contacts, participants were primarily concerned with accuracy, but had different perspectives on which approach would lead to more accurate information. Although most participants thought technology would provide better accuracy, seven participants felt human tracers would be able to elicit more detailed information when identifying contacts through conversation. Participants believed human tracers probe people's memories during the interview: "it is very important to have someone doing the tracing that can follow up on vague answers or prod people with lazy memories and a multitude of other things that an app cannot do" (P195). Additionally, six participants felt that human tracers were better able to clearly ask questions and avoid misunderstanding, which could also result in better quality data: "Allow for more depth in data collection and provide total understanding. Able to probe for explanation of responses." (P64). P172 added that doing so would "Get to speak face to face with human and cut down on any misunderstandings", though we note that human tracing would typically be done through phone calls in the U.S..

5.2.2 Touchpoint 2: Notifying. When being notified, 11 participants expressed fearing inhumane interaction with technology because it could send "incredibly impersonal and insensitive" (P93) messages, especially when people could be "stressed, anxious and worried" (P59) when receiving "such heavy information" (P32) that they have been in contact with someone who tested positive. For example, P93 imagined how a notification received from technology might sound: "hearing from a person is always preferable to receiving a pre-recorded message saying, 'You're screwed.' In a situation like this, the potential for a little empathy is key." Participants felt that technology, on the other hand, can bring convenience when notifying close contacts. Besides supporting automation and scalability, participants appreciated that it would require less effort to receive an app notification or text message than needing to speak with a human tracer: "It's quick and simple, especially if you're notified via text message or push notification from an app" (P25). Additionally, participants felt it would be easier to miss the notification from a call than a text message: "because they won't answer phone calls from unknown numbers" (P80). P60 agreed, saying "I am much more likely to pay attention to and notice a text message than a phone call from an unknown number."

5.2.3 Touchpoint 3: Monitoring. Participants felt the biggest benefit of human tracers in the monitoring touchpoint of reporting symptoms was to make people feel a "sense of company during self-isolation" (P30) because they would be able to talk with people: "The user wouldn't feel lonely, as they would be able to communicate with a human" (P66). Participants feared that technology-caused inhumane interactions during self-quarantine would be more frustrating with the circumstances surrounding COVID-19. P201 said, 
"A lot a person will be doing in self-isolation or self-quarantine is looking at a screen. Maybe another impersonal screen would be a problem emotionally during that stressful time" (P201).

Participants felt that human tracers could collect more accurate health data in this touchpoint because they can help people better identify COVID-related symptoms: "They might be able to help you differentiate if a symptom is actually a symptom for COVID or if you are just assigning everything that happens to COVID out of an overabundance of caution" (P13) and "A human tracer could pick up on symptoms or circumstances that an app might miss out on" (P54). Participants were marginally more willing to share their daily health status with a human tracer rather than technology $(Z=1.85$, $p=0.064,95 \% \mathrm{CI}=0.02$ lower- 0.65 higher on a 5 -point Likert scale). However, participants did not indicate a strong preference when reporting mental status such as stress and anxiety $(p=0.22)$.

Overall, participants felt the convenience of technology in this touchpoint outweighed the potential of human tracers for providing emotional company and accuracy because of the need to share health status on a daily basis. Participants worried that they would find it burdensome to engage with a human tracer, and that they would be "bothered by it everyday." (P3). They also felt it would be simpler to log data via an app: "Easy to log information daily in an app" (P144) since they can "input data at my leisure" (P32) and "just click items on an app" (P61).

\section{DISCUSSION}

Overall, participants saw advantages and drawbacks of both human tracing and digital tracing. Frequency of interaction and people's expectations for the role of human tracers varied in different touchpoints, while the accuracy and scalability that technology can provide were always appreciated. These factors influenced participant's preferences for approaches during different touchpoints, with digital tracing being more favored in the monitoring touchpoint and both approaches being equally valued in the identifying and notifying touchpoint, albeit for different reasons. Due to concerns around data security, participants generally felt that they would be more willing to share some specific information with human tracers.

\subsection{Tracking in Public Health Crisis}

6.1.1 Role of Self-Tracking Technology in Public Health Crises. Context appears to influence people's preferred approaches to contact tracing. Opportunities for and limitations of human tracing and digital tracing vary between three main touchpoints of identifying, notifying, and monitoring, with the actions required for interaction in each touchpoint influencing preferences. Identifying and notifying close contacts typically needs to occur once for an individual, whereas monitoring symptoms is a daily activity throughout the course of self-quarantine. Therefore, participants valued technology's convenience more than the human tracer's emotional support and personalized guidance in the monitoring touchpoint.

Participants imagined that human tracers would apply their human touch slightly differently in each touchpoint. In the identifying touchpoint, participants mainly cared about accuracy, as they wanted to avoid missing any close contacts to report as precisely as possible. Participants appreciated that human tracers could probe the positive cases' memories through follow-up or clarification questions, while technology would have to solely rely on people's own efforts. In the notifying touchpoint, participants feared that receiving unexpected bad news of a positive contact via technology could lead to interactions which felt impersonal or inhumane. Hence, they valued the human tracer's abilities to provide empathy, comfort people under stress, and provide personalized guidance.

However, participants expressed a somewhat paradoxical attitude towards their collaboration with human tracers. The complex context of COVID-19 made participants wish for emotional reassurance and necessary help. But on the other hand, they worried that social anxiety and judgment could result from interacting with human tracers. Therefore, different from previous self-tracking tools' aim of facilitating patient-clinician collaboration around tracked data [50], participants appreciated how self-tracking technology in public health crises can potentially help them avoid the necessity of collaboration by opting into a digital-only approach.

Participants felt contact tracing technology's tracking capabilities were particularly beneficial for providing accuracy and scalability. Participants worried that they would be unable to accurately recall their whereabouts and close contacts even when probed by humans, appreciating that technology might be able to track their recent encounters automatically. They also worried that human tracers may not be able to efficiently notify all close contacts of positive cases, especially if outbreaks occurred and local systems were overly taxed. Since human tracers need to manually log information that positive cases and close contacts provide, their constraints align with the drawbacks of manual tracking reported in prior personal informatics literature [19]. Considering the different affordances that human tracers (e.g., emotional reassurance, advice, and security) and technology (e.g., convenience, scalability, and privacy) can provide, and the varied contexts required for contact tracing, a solution that combines both human tracers and technology should be considered.

In addition, our findings on self-tracking technology's role in public health crises help address some research gaps in both Crisis Informatics and Personal Informatics. The role of self-tracking technology like digital tracing in public health crises is similar to social media's role in other crises. It allows individuals to be digital volunteers [76] by contributing their contact and symptom data to public health authorities in a way similar to the 'bottom-up' risk communication between individuals and authorities enabled by social media $[32,87]$. In Personal Informatics, self-tracking technology typically aims to support personal benefit (e.g., self-understanding). During pandemics, the practice of collecting data about one's self evolves to provide community benefit. Previous studies have primarily focused on designing disease surveillance and response systems for public health benefit, but engaged minimally with people's perspectives on the technology [39,73]. This work, alongside other work in personal informatics, suggests that people's perceptions on the burdens, privacy risks, and value gained from self-tracking influence their interest and adoption [37], which then enables and impacts the role technology may play during these crises.

6.1.2 Privacy and Security in Self-tracking in Public Health Crises. When evaluating their willingness to engage with digital contact tracing, participants evaluated the tradeoffs between their personal 
privacy and security and the public health benefits of contact tracing. Participants often saw human tracing as a preferable option for security reasons. However, we also found that participants sometimes felt other factors were more important, such as convenience and accuracy.

Our study also uncovers people's nuanced attitudes toward privacy in contact tracing. Though privacy in self-tracking studies typically refers to informational privacy (e.g., the control of personal information) [3, 41], participants were more concerned about social privacy (e.g., impression management) and interactional privacy (e.g., control of who you want to interact with) in the context of contact tracing [63]. Participants felt human tracing and technology approaches had similar informational privacy concerns because the same data is required to be disclosed. Although privacy and security have been cited as major barriers to implementing digital tracing for COVID-19 in the United States [65], our study suggests that individuals are mostly concerned about social privacy in contact tracing, such as social anxiety and the fear of being judged. Participants reported fearing that human contact tracers would judge their personal behaviors, since they would need to disclose details about their personal life to help identify potential contacts. Participants also felt having to name close contacts would indicate that they had violated "social distancing" protocols promoted or required by public health authorities [74]. This fear aligns with previous studies finding that people quarantined for COVID-19 exposure were more likely to be judged and avoided by their social connections [11]. Digital tracing, on the other hand, presented an opportunity for people to share this information anonymously [6], giving people the ability to control with whom they want to interact and how others perceive them.

Developing and deploying digital tracing inevitably involves various stakeholders including technology companies, public health agencies, and the government at large. Our participants expressed different levels of trust towards these stakeholders considering the possibility of information being used by technology companies or hacked from databases. While in prior studies of self-tracking systems, such as for physical activity, people have often been comfortable with the data they are disclosing to technology companies because they felt such information was not sensitive [3, 41]. Participants tended to trust public health departments and distrust technology companies and the government at large, feeling that human tracers trained by the public health department had authority and data storage managed by the public health department would be safer. This suggests people prefer to entrust technology to the agency most responsible for crisis response and are generally skeptical of the involvement of technology companies or other government agencies. In practice, health departments often undergo cyber-attacks during public health crises [33], potentially suggesting differences between participant's perceived security risks and the actual risks. Therefore, the design of digital tracing should consider what influence various stakeholders in the implementation and rollout of digital tracing might have on public perception of that technology. For example, involved technology companies could erode individuals' trust, although their expertise in creating scalable, reliable, and secure technology could be helpful for containing the pandemic's spread.

\subsection{Design Recommendations}

6.2.1 A Flexible Hybrid System to Involve Both Human Tracing and Technology. As Choe et al. [19] proposed a semi-automated approach for self-tracking combining the strengths of both manual tracking and automated tracking, we propose opportunities for a hybrid system that involves both human tracing and contact tracing technology. Compared with a fully automated system, our participant perspectives suggest benefits for involving human tracing or introducing more conversational or empathic features into digital tracing. As participants saw humans and technology as better suited to addressing different aspects of contact tracing, technology could incorporate human tracing into a single contact tracing approach.

A hybrid system can also acknowledge that preferences are circumstantial to people's abilities, access to technology, and personal preferences. For example, to support people that can reliably access smartphones and the internet, the system could mostly utilize selftracking technology to contact, notify, and interact at scale. Such an approach could still provide convenient access to human tracing, such as enabling users to directly chat with human tracers via text, voice messages, or videos. For those with low technology access or abilities, the system would mainly adopt the human tracing approach to avoid an information gap caused by lacking accessibility to the technology. However, a reasonable concern is that hybrid solutions could introduce inequities in care if some people could be traced more accurately or contacted more efficiently [4].

We suggest that digital tracing could be more humane, showing more care to individuals who are suffering from the pandemic. Technology companies have provided AI chatbots to facilitate contact tracing by providing tailored communication [23] and Virginia's contact tracing app has included a virtual chatbot feature [31]. Previous work [56] has shown that health chatbots are able to provide empathy while also protecting end-users' privacy by not sharing information with the chatbot's creator. Therefore, a hybrid contact tracing system could utilize chatbots to augment human tracing, providing emotional support to broader populations given finite human resources. An AI-powered chatbot has the potential to be used at scale by engaging in empathic communication with numerous end-users during self-quarantine while protecting their privacy. It can also connect people with necessary resources, such as websites of public health departments and Question \& Answer (Q\&A) sections about COVID-19. In the notifying touchpoint, a hybrid contact tracing app could leverage technology to accurately notify close contacts at scale, but leverage humans through an in-app chat or provide a hotline to call for advice and emotional support roles when close contacts received unexpected bad news, instead of simply utilizing virtual chatbot or notification message in the app.

To provide a more caring experience, some common design solutions utilized in personal tracking tools could be applied to contact tracing systems. For example, self-tracking applications have frequently leveraged social support, even in anonymous communities, to connect users who are tracking similar data to share their experiences and struggles [54]. Moreover, instead of simply collecting people's health information to monitor it, contact tracing technology could also promote self-understanding by providing reports based on the symptoms people log to help them understand their condition. Technology such as CovidNearYou [49] and the COVID 
symptom study app [28] have not only supported collecting personal data for researcher understanding, but also enabled individuals to better understand their risk levels by visualizing hotspots in their surroundings collected from other's data. By seeing one's contribution in a public space, these apps also convey that individual's efforts are valued for public health.

6.2.2 Building Trust Through Authority and Transparency. Considering how participants perceived different stakeholders often involved in developing and deploying digital tracing, we suggest digital tracing can leverage a combination of authority provided by public health departments and transparency around how tracing systems practically work. For example, contact tracing technology could indicate what government agencies or private companies will have access to information provided, and to what data stored in the technology each will have access. Contact tracing technology created in consultation with public health agencies could also leverage its authority to convey public health guidelines by incorporating Q\&A's or other recommendations often provided on the agency's websites. Technology could guide people to specific questions or topics in response to their current circumstances (e.g., if they have been identified as a close contact of a confirmed case) or reacting to data they $\log$ (e.g., describing what to do upon logging potential symptoms).

\section{CONCLUSION}

In examining people's perceptions of human and technology approaches to contact tracing, we identify respective strengths and weaknesses of each approach. The specific touchpoint of contact tracing influences how people weigh the personalized interaction a human tracer can provide versus the convenience that automatic and manual tracking features in technology can provide. We suggest that hybrid contact tracing systems can integrate both approaches to suit people's different backgrounds and needs, introducing conversational or self-understanding elements to make technology appear more humane. Increasing transparency around how data is stored and who has access and leveraging the authority of public health systems can further help build trust in contact tracing solutions.

\section{ACKNOWLEDGMENTS}

We thank Mayara Costa Figueiredo, Lucas de Melo Silva, Naomi Yamashita, and our anonymous reviewers for their feedback. This work was funded in part by the National Science Foundation under award IIS-1850389.

\section{REFERENCES}

[1] Aarogya Setu: Why India's Covid-19 Contact Tracing App is Controversial - BBC News. https://www.bbc.com/news/world-asia-india-52659520

[2] Johannes Abeler, Matthias Bäcker, Ulf Buermeyer, and Hannah Zillessen. (2020) Covid-19 Contact Tracing and Data Protection can Go Together. 7MIR mHealth and uHealth 8, e19359. http://doi.org/10.2196/19359

[3] Angeliki Aktypi, Jason R C Nurse, and Michael Goldsmith. (2017). Unwinding Ariadne's Identity Thread: Privacy Risks with Fitness Trackers and Online Social Networks. Proceedings of the 2017 on Multimedia Privacy and Security - MPS '17, ACM Press. https://doi.org/10.1145/3137616.3137617

[4] Ali Alkhatib. (2020). We Need to Talk about Digital Contact Tracing. Interactions, 27(4), 84-89. http://doi.org/10.1145/3404205

[5] Tim Althoff, Rok Sosič, Jennifer L. Hicks, Abby C. King, Scott L. Delp, and Jure Leskovec. (2017). Large-Scale Physical Activity Data Reveal Worldwide Activity Inequality. Nature, 547(7663), 336-339. http://doi.org/10.1038/nature23018
[6] Apple and Google Release Sample Code for Contract Tracing Apps. https://www.cnbc.com/2020/05/04/apple-and-google-release-sample-code-forcontract-tracing-apps.html

[7] Kristin Baltrusaitis, John S. Brownstein, Samuel V. Scarpino, Eric Bakota, Adam W. Crawley, Giuseppe Conidi, Julia Gunn, Josh Gray, Anna Zink, and Mauricio Santillana. (2018). Comparison of Crowd-Sourced, Electronic Health Records Based, and Traditional Health-Care Based Influenza-Tracking Systems at Multiple Spatial Resolutions in the United States of America. BMC Infectious Diseases, 18(1), 403. http://doi.org/10.1186/s12879-018-3322-3

[8] Bluetooth Signals From Your Smartphone Could Automate Covid-19 Contact Tracing While Preserving Privacy | MIT News | Massachusetts Institute of Technology. https://news.mit.edu/2020/bluetooth-covid-19-contact-tracing-0409

[9] Isobel Braithwaite, Thomas Callender, Miriam Bullock, and Robert W Aldridge. (2020). Automated and Partly Automated Contact Tracing: A Systematic Review to Inform the Control of COVID-19. The Lancet Digital Health, O(0). http://doi. org/10.1016/s2589-7500(20)30184-9

[10] Virginia Braun and Victoria Clarke. (2012). Thematic Analysis. In APA handbook of research methods in psychology, Vol 2: Research designs: Quantitative, qualitative, neuropsychological, and biological. American Psychological Association, 57-71. http://doi.org/10.1037/13620-004

[11] Samantha K. Brooks, Rebecca K. Webster, Louise E. Smith, Lisa Woodland, Simon Wessely, Neil Greenberg, and Gideon James Rubin. (2020). The Psychological Impact of Quarantine and How to Reduce it: Rapid Review of the Evidence. The Lancet 395, 912-920. http://doi.org/10.1016/S0140-6736(20)30460-8

[12] Vir B. Bulchandani, Saumya Shivam, Sanjay Moudgalya, and S. L. Sondhi. (2020). Digital Herd Immunity and COVID-19. http://arxiv.org/abs/2004.07237

[13] Can Asymptomatic Patients Spread Coronavirus? Here's What a New Study Reveals. | Advisory Board Daily Briefing. https://www.advisory.com/daily-briefing/ 2020/08/10/asymptomatic

[14] Marco Cascella, Michael Rajnik, Arturo Cuomo, Scott C. Dulebohn, and Raffaela Di Napoli. (2020). Features, Evaluation and Treatment Coronavirus (COVID-19). StatPearls Publishing. http://www.ncbi.nlm.nih.gov/pubmed/32150360

[15] Case Investigation and Contact Tracing: Part of a Multipronged Approach to Fight the COVID-19 Pandemic | CDC. https://www.cdc.gov/coronavirus/2019ncov/php/principles-contact-tracing.html

[16] CDC - BRFSS - Questionnaires. https://www.cdc.gov/brfss/questionnaires/index. htm

[17] CDC and Ncird. Guidelines for the Implementation and Use of Digital Tools to A ugment Traditional Contact Tracing COVID-19 Contact Tracing for Health Departments.

[18] Apoorva Chauhan and Amanda Lee Hughes. (2017). Providing Online Crisis Information: An Analysis of Official Sources during the 2014 Carlton Complex Wildfire. Conference on Human Factors in Computing Systems - Proceedings, Association for Computing Machinery, 3151-3162. http://doi.org/10.1145/3025453.3025627

[19] Eun Kyoung Choe, Saeed Abdullah, Mashfiqui Rabbi, Edison Thomaz, Daniel A. Epstein, Felicia Cordeiro, Matthew Kay, Gregory D. Abowd, Tanzeem Choudhury, James Fogarty, Bongshin Lee, Mark Matthews, and Julie A. Kientz. (2017). SemiAutomated Tracking: A Balanced Approach for Self-Monitoring Applications. IEEE Pervasive Computing, 16(1), 74-84. http://doi.org/10.1109/MPRV.2017.18

[20] Contact-Tracing Apps are Political. https://www.brookings.edu/techstream/ contact-tracing-apps-are-political/

[21] Contact tracing - Coronavirus COVID-19 Response. https://covid19.ca.gov/ contact-tracing/

[22] Contact Tracing Apps Now Cover Nearly Half of America. It's Not Too Late to Use One. | MIT Technology Review. https://www.technologyreview.com/2020/ 12/14/1014426/covid-california-contact-tracing-app-america-states/

[23] Contact Tracing from Avaya | Leverage Automation and AI Avaya. $\quad \mathrm{https}$ //www.avaya.com/en/contact-tracing/?CTA=20USCC-DG-CTTRC-SEM\&TAC $=20$ US-CC-DG-CTTRC-SEM\&gclid= Cj0KCQjwqfz6BRD8ARIsAIXQCf0wS6b9P8SiZiDYalVflbOCQHYk2VyPITRQ7K1d 9pvCC5IlQoHLUMaApaQEALw_wcB

[24] Coronavirus (COVID-19) Frequently Asked Questions | CDC. https://www.cdc. gov/coronavirus/2019-ncov/faq.html\#Contact-Tracing

[25] Country Spotlight: Singapore's TraceTogether Program | Berkman Klein Center. https://cyber.harvard.edu/story/2020-07/country-spotlight-singaporestracetogether-program

[26] COVID-19 Contact Tracing | CDC. https://www.cdc.gov/coronavirus/2019-ncov/ daily-life-coping/contact-tracing.html

[27] COVID-19 Contact Tracing Apps Reach 9\% Adoption In Most Populous Countries. https://sensortower.com/blog/contact-tracing-app-adoption

[28] COVID Symptom Study - Help Slow the Spread of COVID-19. https://covid. joinzoe.com/us- 2

[29] COVIDSafe app | Australian Government Department of Health. https://www. health.gov.au/resources/apps-and-tools/covidsafe-app

[30] COVIDWISE App Tops 300,000 Downloads. https://www.nbc12.com/2020/08/12/ covidwise-app-tops-downloads/

[31] COVIDWISE on the App Store. https://apps.apple.com/us/app/id1518059690 
[32] Crisis Informatics Research Literature Resource - Google Docs. https://docs. google.com/document/d/1g6I8Br3vTC04iXVHFTee6arKVld89x9bGvSEeN_ $\mathrm{NaPU} / \mathrm{edit} \#$

[33] Cyberattack Hits HHS During Coronavirus Response - Bloomberg. https://www.bloomberg.com/news/articles/2020-03-16/u-s-health-agencysuffers-cyber-attack-during-covid-19-response

[34] Digital Tools for COVID-19 Contact Tracing. https://www.who.int/publications/ i/item/WHO-2019-nCoV-Contact Tracing-Tools Annex-2020.1

[35] Ensheng Dong, Hongru Du, and Lauren Gardner. (2020). An Interactive WebBased Dashboard to Track COVID-19 in Real Time. The Lancet Infectious Diseases 20, 533-534. http://doi.org/10.1016/S1473-3099(20)30120-1

[36] Daniel A. Epstein, Clara Caldeira, Mayara Costa Figueiredo, Xi Lu, Lucas M. Silva, Lucretia Williams, Jong Ho Lee, Qingyang Li, Simran Ahuja, Qiuer Chen, Payam Dowlatyari, Craig Hilby, Sazeda Sultana, Elizabeth V. Eikey, and Yunan Chen. (2020). Mapping and Taking Stock of the Personal Informatics Literature. Proceedings of the ACM on Interactive, Mobile, Wearable and Ubiquitous Technologies, 4(4), 1-38. http://doi.org/10.1145/3432231

[37] Daniel A. Epstein, Monica Caraway, Chuck Johnston, An Ping, James Fogarty, and Sean A. Munson. (2016). Beyond Abandonment to Next Steps: Understanding and Designing for Life After Personal Informatics Tool Use. Proceedings of the SIGCHI Conference on Human Factors in Computing Systems (CHI 2016), 1109-1113. https://doi.org/10.1145/2858036.2858045

[38] Luca Ferretti, Chris Wymant, Michelle Kendall, Lele Zhao, Anel Nurtay, Lucie Abeler-Dörner, Michael Parker, David Bonsall, and Christophe Fraser. (2020) Quantifying SARS-CoV-2 Transmission Suggests Epidemic Control with Digital Contact Tracing. Science, 368(6491). http://doi.org/10.1126/science.abb6936

[39] Clark C. Freifeld, Rumi Chunara, Sumiko R. Mekaru, Emily H. Chan, Taha KassHout, Anahi Ayala Iacucci, and John S. Brownstein. (2010). Participatory Epidemiology: Use of Mobile Phones for Community-Based Health Reporting. PLoS Medicine, 7(12), e1000376. http://doi.org/10.1371/journal.pmed.1000376

[40] Vicki S. Freimuth. (2006). Order Out of Chaos: The Self-Organization of Communication Following the Anthrax Attacks. Health Communication 20, 141-148. http://doi.org/10.1207/s15327027hc2002 5

[41] Sandra Gabriele and Sonia Chiasson. (2020). Understanding Fitness Tracker Users Security and Privacy Knowledge, Attitudes and Behaviours. Proceedings of the 2020 CHI Conference on Human Factors in Computing Systems, Association for Computing Machinery (ACM), 1-12. http://doi.org/10.1145/3313831.3376651

[42] Urs Gasser, Marcello Ienca, James Scheibner, Joanna Sleigh, and Effy Vayena (2020). Digital Tools against COVID-19: Taxonomy, Ethical challenges, and Navigation aid. The Lancet Digital Health 2, e425-e434. http://doi.org/10.1016/S25897500(20)30137-0

[43] Mitchell L. Gordon, Tim Althoff, and Jure Leskovec. (2019). Goal-Setting and Achievement in Activity Tracking Apps: A case study of MyFitnessPal. The Web Conference 2019 - Proceedings of the World Wide Web Conference, WWW 2019, Association for Computing Machinery, Inc, 571-582. http://doi.org/10.1145/ 3308558.3313432

[44] Xinning Gui, Yubo Kou, Kathleen H. Pine, and Yunan Chen. (2017). Managing Uncertainty: Using Social Media for Risk Assessment during a Public Health Crisis. Conference on Human Factors in Computing Systems - Proceedings, Association for Computing Machinery, 4520-4533. http://doi.org/10.1145/3025453.3025891

[45] Xinning Gui, Yubo Kou, Kathleen Pine, Elisa Ladaw, Harold Kim, Eli SuzukiGill, and Yunan Chen. (2018). Multidimensional Risk Communication: Public Discourse on Risks During an Emerging Epidemic. Conference on Human Factors in Computing Systems - Proceedings, Association for Computing Machinery, 1-14. http://doi.org/10.1145/3173574.3173788

[46] GuideSafe on the App Store. https://apps.apple.com/us/app/guidesafe/ id1519514691

[47] Rajan Gupta, Manan Bedi, Prashi Goyal, Srishti Wadhera, and Vaishnavi Verma. (2020). Analysis of COVID-19 Tracking Tool in India: Case Study of Aarogya Setu Mobile Application. Digital Government: Research and Practice, 1(4), 1-8. http://doi.org/10.1145/3416088

[48] Cassandra Harrison, Mohip Jorder, Henri Stern, Faina Stavinsky, Vasudha Reddy, Heather Hanson, HaeNa Waechter, Luther Lowe, Luis Gravano, Sharon Balter, and Centers for Disease Control and Prevention (CDC). (2014). Using Online Reviews by Restaurant Patrons to Identify Unreported Cases of Foodborne Illness - New York City, 2012-2013. MMWR. Morbidity and mortality weekly report, 63(20), 441-5. http://www.ncbi.nlm.nih.gov/pubmed/24848215

[49] Home | Covid Near You. https://covidnearyou.org/us/en-US

[50] Matthew K. Hong, Udaya Lakshmi, Thomas A. Olson, and Lauren Wilcox. (2018). Visual ODLs: Co-Designing Patient-Generated Observations of Daily Living to Support Data-Driven Conversations in Pediatric Care. Conference on Human Factors in Computing Systems - Proceedings, Association for Computing Machinery, 1-13. http://doi.org/10.1145/3173574.3174050

[51] How Digital Contact Tracing Slowed Covid-19 in East Asia. https://hbr.org/2020/ 04/how-digital-contact-tracing-slowed-covid-19-in-east-asia

[52] How Long Will Coronavirus Last? Pandemic Will Stretch Into 2021 Bloomberg. https://www.bloomberg.com/news/features/2020-06-18/we-will-be- living-with-the-coronavirus-pandemic-well-into-2021

[53] How to Stop the Stigma That's Spreading with COVID-19| South Shore Health. https://www.southshorehealth.org/wellness/blog/how-stop-stigma-thatsspreading-covid-19

[54] Heewon Kim, Meara Faw, and Andreas Michaelides. (2017). Mobile But Connected: Harnessing the Power of Self-Efficacy and Group Support for Weight Loss Success through mHealth Intervention. Journal of Health Communication, 22(5), 395-402. http://doi.org/10.1080/10810730.2017.1296510

[55] Young-Ho Kim, Jae Ho Jeon, Bongshin Lee, Eun Kyoung Choe, and Jinwook Seo. (2017). OmniTrack: A Flexible Self-Tracking Approach Leveraging SemiAutomated Tracking. Proceedings of the ACM on Interactive, Mobile, Wearable and Ubiquitous Technologies, 1(3), 1-28. http://doi.org/10.1145/3130930

[56] Rafal Kocielnik, Elena Agapie, Alexander Argyle, Dennis T. Hsieh, Kabir Yadav, Breena Taira, and Gary Hsieh. (2019). HarborBot: A Chatbot for Social Needs Screening. AMIA ... Annual Symposium proceedings. AMIA Symposium, 2019, 552-561. https://botui.org/

[57] Leslie Lenert and Brooke Yeager McSwain. (2020). Balancing Health Privacy, Health Information Exchange, and Research in the Context of the COVID-19 Pandemic. Journal of the American Medical Informatics Association 27, 963-966. http://doi.org/10.1093/jamia/ocaa039

[58] Ian Li, Anind Dey, and Jodi Forlizzi. (2010). A Stage-based Model of Personal Informatics Systems. Proceedings of the SIGCHI Conference on Human Factors in Computing Systems (CHI 2010), 557-566. https://doi.org/10.1145/1753326.1753409

[59] Life as a Covid-19 Contact Tracer: Sleuthing, Stress, Going Off-Script - STAT. https://www.statnews.com/2020/05/18/coronavirus-contact-tracer-sleuthingstress-veering-off-script/

[60] Brian Y. Lim, Judy Kay, and Weilong Liu. (2019). How Does a Nation Walk? Interpreting Large-Scale Step Count Activity with Weekly Streak Patterns. Proceedings of the ACM on Interactive, Mobile, Wearable and Ubiquitous Technologies, 3(2), 1-46. http://doi.org/10.1145/3328928

[61] Mobile Tracking and Privacy in the Coronavirus Pandemic | ACM Interactions. https://interactions.acm.org/archive/view/july-august-2020/mobiletracking-and-privacy-in-the-coronavirus-pandemic?doi=10.1145\%2F3404121

[62] New York City Partnering with Twilio for Contact Tracing Initiative - TechRepublic. https://www.techrepublic.com/article/new-york-city-partnering-withtwilio-for-contact-tracing-initiative/

[63] Xinru Page, Alfred Kobsa, and Bart P Knijnenburg. (2012). Don't Disturb My Circles! Boundary Preservation Is at the Center of Location-Sharing Concerns. Proceedings of the Thirteenth International AAAI Conference on Web and Social Media. https://www.ics.uci.edu/ kobsa/papers/2012-ICWSM-kobsa.pdf

[64] Leysia Palen and Sophia B. Liu. (2007). Citizen Communications in Crisis: Anticipating a Future of ICT-Supported Public Participation. Conference on $\mathrm{Hu}$ man Factors in Computing Systems - Proceedings, ACM Press, 727-736. http: //doi.org/10.1145/1240624.1240736

[65] Sangchul Park, Gina Jeehyun Choi, and Haksoo Ko. (2020). Information Technology-Based Tracing Strategy in Response to COVID-19 in South Korea - Privacy Controversies. JAMA - Fournal of the American Medical Association, 323(21), 2129-2130. http://doi.org/10.1001/jama.2020.6602

[66] Prolific | Online Participant Recruitment for Surveys and Market Research. https: //www.prolific.co/

[67] Qualtrics XM // The Leading Experience Management Software. https://www. qualtrics.com/

[68] Roundup: Tech's Role in Tracking, Testing, Treating COVID-19| MobiHealthNews. https://www.mobihealthnews.com/news/roundup-techs-role-trackingtesting-treating-covid-19

[69] Jilian A. Sacks, Elizabeth Zehe, Cindil Redick, Alhoussaine Bah, Kai Cowger, Mamady Camara, Aboubacar DIallo, Abdel Nasser Iro Gigo, Ranu S. Dhillon, and Anne Liu. (2015). Introduction of Mobile Health Tools to Support Ebola Surveillance and Contact Tracing in Guinea. Global Health Science and Practice, 3(4), 646-659. http://doi.org/10.9745/GHSP-D-15-00207

[70] Ali Shameli, Tim Althoff, Amin Saberi, and Jure Leskovec. (2019). How Gamification Affects Physical Activity: Large-scale Analysis of Walking Challenges in a Mobile Application. 26th International World Wide Web Conference 2017, WWW 2017 Companion, International World Wide Web Conferences Steering Committee, 455-463. http://doi.org/10.1145/3041021.3054172

[71] Singapore Government Launches New App for Contact Tracing to Combat Spread of COVID-19 | MobiHealthNews. https://www.mobihealthnews.com/news/asiapacific/singapore-government-launches-new-app-contact-tracing-combatspread-covid-19

[72] Singapore Says COVID-19 Contact-Tracing Data Can Be Requested By Police: Coronavirus Updates: NPR. https://www.npr.org/sections/coronavirus-liveupdates/2021/01/05/953604553/singapore-says-covid-19-contact-tracing-datacan-be-requested-by-police

[73] Mark S. Smolinski, Adam W. Crawley, Kristin Baltrusaitis, Rumi Chunara, Jennifer M. Olsen, Oktawia Wójcik, Mauricio Santillana, Andre Nguyen, and John S. Brownstein. (2015). Flu near you: Crowdsourced symptom reporting spanning 2 influenza seasons. American fournal of Public Health, 105(10), 2124-2130. http: //doi.org/10.2105/AJPH.2015.302696 
[74] Social Distancing, Ouarantine, and Isolation. https://www.cdc.gov/coronavirus/ 2019-ncov/prevent-getting-sick/social-distancing.html

[75] South Korea's Widespread Testing And Contact Tracing Lead To First Day With No New Cases. https://www.forbes.com/sites/alexandrasternlicht/2020/04/30/ south-koreas-widespread-testing-and-contact-tracing-lead-to-first-day-withno-new-cases/\#7013fa8c5abf

[76] Kate Starbird and Leysia Palen. (2011). "Voluntweeters": Self-Organizing by Digital Volunteers in Times of Crisis. Conference on Human Factors in Computing Systems - Proceedings, ACM Press, 1071-1080. http://doi.org/10.1145/1978942.1979102

[77] State Approaches to Contact Tracing during the COVID-19 Pandemic - The National Academy for State Health Policy. https://www.nashp.org/state-approachesto-contact-tracing-covid-19/

[78] "The First Day was Really Hard": Life as A Contact Tracer | MIT Technology Review. https://www.technologyreview.com/2020/05/10/1001534/first-personamerica-covid-19-contact-tracer-experience/

[79] Zachary O. Toups and Andruid Kerne. (2007). Implicit Coordination in Fire fighting Practice: Design Implications for Teaching Fire Emergency Responders Conference on Human Factors in Computing Systems - Proceedings, ACM Press, 707-716. http://doi.org/10.1145/1240624.1240734

[80] U.S. Census Bureau QuickFacts: United States. https://www.census.gov/ quickfacts/fact/table/US/PST045219

[81] Urban Residents in States Hit Hard by COVID-19 Most Likely to See it as a Threat to Daily Life | Pew Research Center. https://www.pewresearch.org/facttank/2020/03/20/urban-residents-in-states-hit-hard-by-covid-19-most-likelyto-see-it-as-a-threat-to-daily-life/
[82] Sarah Vieweg, Amanda L. Hughes, Kate Starbird, and Leysia Palen. (2010). Microblogging during Two Natural Hazards Events: What Twitter may Contribute to Situational Awareness. Conference on Human Factors in Computing Systems Proceedings, ACM Press, 1079-1088. http://doi.org/10.1145/1753326.1753486

[83] Virginia Becomes First State to Try Covidwise Pandemic App From Apple, Google - NBC4 Washington. https://www.nbcwashington.com/news/health/ virginia-becomes-first-state-to-try-covidwise-pandemic-app-from-applegoogle/2382430/

[84] Sera Whitelaw, Mamas A. Mamas, Eric Topol, and Harriette G.C. Van Spall. (2020). Applications of Digital Technology in COVID-19 Pandemic Planning and Response. The Lancet Digital Health 2, e435-e440. http://doi.org/10.1016/S25897500(20)30142-4

[85] Will Americans Be Willing to Install COVID-19 Tracking Apps? - Scientific American Blog Network. https://blogs.scientificamerican.com/observations/willamericans-be-willing-to-install-covid-19-tracking-apps/

[86] Christopher Wilson and Maria Gabrielsen Jumbert. (2018). The New Informatics of Pandemic Response: Humanitarian Technology, Efficiency, and the Subtle Retreat of National Agency. Journal of International Humanitarian Action, 3(1), 8. http://doi.org/10.1186/s41018-018-0036-5

[87] Yixuan Zhang, Nurul Suhaimi, Rana Azghandi, Mary Amulya Joseph, Miso Kim, Jacqueline Griffin, and Andrea G. Parker. (2020). Understanding the Use of Crisis Informatics Technology among Older Adults. Proceedings of the $2020 \mathrm{CHI}$ Conference on Human Factors in Computing Systems, Association for Computing Machinery (ACM), 1-13. http://doi.org/10.1145/3313831.3376862 